\title{
Tidal Effects in the Post-Minkowskian Expansion
}

\author{
Clifford Cheung and Mikhail P. Solon \\ Walter Burke Institute for Theoretical Physics, California Institute of Technology, Pasadena, California 91125
}

(Received 26 June 2020; accepted 30 September 2020; published 2 November 2020)

\begin{abstract}
Tools from scattering amplitudes and effective field theory have recently been repurposed to derive stateof-the-art results for the black hole binary inspiral in the post-Minkowskian expansion. In the present Letter, we extend this approach to include the tidal effects of mass and current quadrupoles on the conservative dynamics of nonspinning neutron star mergers. We compute the leading and, for the first time, next-to-leading order post-Minkowskian finite size corrections to the conservative Hamiltonian, together with their associated scattering amplitudes and scattering angles. Our expressions are gauge invariant and, in the extreme mass ratio limit, consistent with the dynamics of a tidally deformed test body in a Schwarzschild background. Furthermore, they agree completely with existing results at leading postMinkowskian and second post-Newtonian orders.
\end{abstract}

DOI: 10.1103/PhysRevLett.125.191601

Introduction.-The monumental discovery of gravitational waves by LIGO and Virgo [1] has sparked a flurry of activity in applying ideas from the study of scattering amplitudes and effective field theory (EFT) to the binary inspiral problem. Building on seminal work on the quantum field theoretic description of gravitons [2,3], this nascent program has fused cutting edge tools from the double copy [4], generalized unitarity [5], and EFT [6,7] to obtain the now state-of-the-art $\mathcal{O}\left(G^{3}\right)$ conservative Hamiltonian for spinless binary black holes [8-10].

New results have also been derived for binary systems with spin [11,12], with supersymmetry [13], and for scattering of massless particles with or without supersymmetry [14]. Other advances in this area have utilized classic tools from quantum mechanics and quantum field theory $[15,16]$, newly uncovered amplitudes structures [17], and analytic continuation between the scattering and bound state problems [18].

There is, of course, an illustrious record of tackling this subject with conventional methods, such as effective onebody formalism [19], numerical relativity [20], the self-force formalism [21], and perturbative analysis using post-Newtonian (PN) [22], post-Minkowskian (PM) [23], and nonrelativistic general relativity $[3,24]$.

Importantly, these more traditional approaches have all been adapted to a principal scientific aim of the gravitational wave program: disentangling the underlying nuclear properties of neutron stars (see Refs. $[25,26]$ for reviews).

Published by the American Physical Society under the terms of the Creative Commons Attribution 4.0 International license. Further distribution of this work must maintain attribution to the author(s) and the published article's title, journal citation, and DOI. Funded by SCOAP.
Recent detections of gravitational waves generated by the inspiral and merger of neutron stars have already put direct constraints on the equation of state of matter at nuclear densities [27,28], and much effort has been dedicated to the prospects and challenges for maximizing the science yield from current and future measurements [29,30]. Tidal effects have been modeled using a variety of numerical [31] and analytic methods [29,32-34], including the self-force [35] and effective one-body [36] formalisms and, very recently, using PM perturbation theory $[37,38]$. These, together with the prospect of accurate measurements of tidal parameters at future third-generation detectors like the Einstein Telescope [39], all offer strong motivations to extend the tools of scattering amplitudes and EFT to incorporate the corresponding finite size effects.

In this Letter, we compute the leading and next-toleading PM conservative Hamiltonian induced by the mass and current quadrupole moments of spinless compact bodies. To begin, we compute Feynman diagrams describing the scattering of nonminimally coupled, gravitationally interacting massive scalars at one- and two-loop orders in a general field basis and gauge fixing. These objects encode the leading $\mathcal{O}\left(G^{2}\right)$ and next-to-leading $\mathcal{O}\left(G^{3}\right)$ PM tidal corrections. We then integrate these Feynman diagrams via the methods of [7,9] to obtain a gauge invariant scattering amplitude. Equating this to the amplitude computed in a nonrelativistic EFT, we then derive the corresponding conservative Hamiltonian for tidal effects. Throughout, we work at linear order in the tidal coefficients while resumming to all orders in the velocity expansion.

As a check, we compute the associated scattering angle and find exact agreement with the leading order PM results of $[37,38]$. At the relevant overlapping orders, our expressions are also consistent with existing results for the 2PN Hamiltonian and 1PN binding energy [34,36]. Last but not 
least, the test-particle limit of our Hamiltonian is gauge equivalent to that of a tidally deformed test mass in a Schwarzschild background.

Setup.-Our setup is described by a pair of massive scalars interacting gravitationally via minimal coupling (we work in mostly plus metric signature throughout),

$S=\int d^{4} x \sqrt{-g}\left(\frac{R}{16 \pi G}-\frac{1}{2} \sum_{i=1,2}\left(\nabla^{\mu} \phi_{i} \nabla_{\mu} \phi_{i}+m_{i}^{2} \phi_{i}^{2}\right)\right)$,

together with additional higher dimension operators encoding tidal distortions,

$$
\begin{aligned}
\Delta S= & \int d^{4} x \sqrt{-g} \frac{1}{4} C_{\mu \alpha \nu \beta} C^{\rho \alpha \sigma \beta} \\
& \times \sum_{i=1,2}\left(\lambda_{i} \phi_{i}^{2} \delta_{\rho}^{\mu} \delta_{\sigma}^{\nu}+\frac{\eta_{i}}{m_{i}^{4}} \nabla^{\mu} \nabla^{\nu} \phi_{i} \nabla_{\rho} \nabla_{\sigma} \phi_{i}\right) .
\end{aligned}
$$

The coefficients $\lambda_{i}$ and $\eta_{i}$ are couplings of mass dimension minus two, which parametrize linear combinations of the mass and current quadrupoles. We set $\lambda_{1}, \eta_{1} \neq 0$ and $\lambda_{2}=\eta_{2}=0$, and the general case is trivially obtained by symmetrizing over particle labels. Hereafter, all variables with a $\Delta$ prefix will denote quantities linear in the tidal coefficients. For an explicit mapping between the operators above and the standard worldline formalism, see Eqs. (11) and (12).

Note that higher dimension operators with more than four derivatives on the scalars require additional derivatives on the gravitational field and thus describe higher order tidal moments. All other allowed operators can be eliminated either through field redefinitions or Weyl tensor identities, such as $C_{\alpha \beta \gamma}^{\mu} C^{\nu \alpha \beta \gamma}=\frac{1}{4} g^{\mu \nu} C_{\alpha \beta \gamma \delta} C^{\alpha \beta \gamma \delta}$ [40], however, they are straightforwardly included as a consistency check of the calculation.

Scattering amplitudes.-In this section, we compute the leading and next-to-leading PM tidal corrections to scattering, $\Delta M_{2}$ and $\Delta M_{3}$. As discussed at length in [9], all diagrams with self-energy loops or contact interactions do not contribute classically. The relevant one- and two-loop Feynman diagrams are depicted in Fig. 1.

We perform our entire calculation in the generalized graviton field basis and gauge fixing described in [10], utilized previously to simplify perturbation theory [41] and containing the de Donder gauge as a subset. As a highly nontrivial consistency check, all gauge dependence will vanish from the physical amplitude. Hereafter, any gaugedependent expressions will be in de Donder gauge.

As described in [9], the cumbersome multiloop integrands computed using Feynman diagrams can be massively simplified by applying a procedure for classical truncation, which eliminates quantum corrections at the integrand level. Operationally, this is achieved by a series

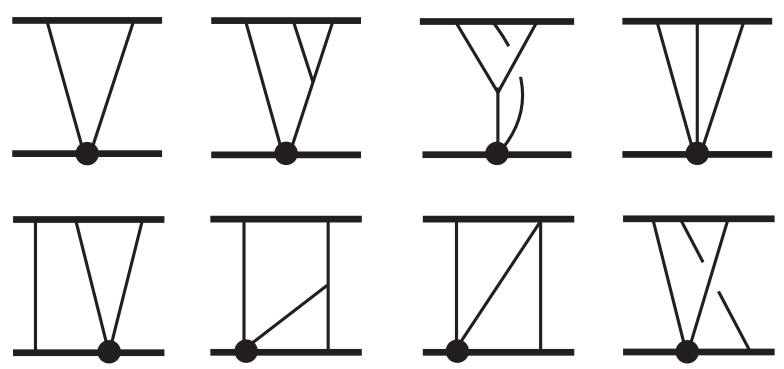

FIG. 1. Feynman diagrams for classical tidal corrections. The thick and thin lines denote massive scalars and exchanged gravitons, respectively, while black circles are tidal operator insertions. Not shown are "reflected" graphs obtained by swapping the scalars, nor "twisted" graphs obtained by swapping the incoming and outgoing legs for one of the scalars.

expansion in small $\epsilon$ following the replacement $q, \ell \rightarrow \epsilon q, \epsilon \ell$, where $q$ is the four-momentum transfer and $\ell$ is any graviton loop four-momentum. The series in $\epsilon \sim q / p_{1,2} \sim \hbar / J$ for incoming four-momenta $p_{1,2}$ is an expansion in large angular momentum. The classical contributions to the amplitude at $\mathcal{O}\left(G^{n}\right)$ scale as $M_{n} \rightarrow$ $\epsilon^{n-3} M_{n}$ and $\Delta M_{n} \rightarrow \epsilon^{n+1} \Delta M_{n}$, modulo infrared divergent "iteration" terms that are lower order in $\epsilon$ and appear exactly in the EFT in such a way that cancels in the matching to the Hamiltonian. (The difference of $\epsilon^{4}$ scaling between $M_{n}$ and $\Delta M_{n}$ follow from four additional derivatives with respect to graviton momenta in the curvature squared couplings.)

At one loop there is a single "triangle" Feynman diagram, shown in Fig. 1, that survives classical truncation. The corresponding integrand is

$$
\begin{aligned}
\Delta I_{2}= & -\frac{32 G^{2} \pi^{2} m_{2}^{4} \boldsymbol{q}^{4}}{\ell^{2}(\ell+q)^{2}\left(\ell^{2}+2 p_{2} \ell\right)}\left[4 \lambda_{1}+\frac{\eta_{1}}{2}\left(\left(1-2 \sigma^{2}\right)^{2}\right.\right. \\
& \left.\left.-\frac{4\left(p_{1} \ell\right)^{2}}{m_{1}^{2} \boldsymbol{q}^{2}}\left(1-4 \sigma^{2}\right)+\frac{8\left(p_{1} \ell\right)^{4}}{m_{1}^{4} \boldsymbol{q}^{4}}\right)\right]
\end{aligned}
$$

where $\sigma=-p_{1} p_{2} / m_{1} m_{2}$. We then evaluate the integral $\Delta M_{2}=\int\left[d^{4} \ell /(2 \pi)^{4}\right] \Delta I_{2}$ via standard relativistic methods or via the nonrelativistic approach in [9], obtaining

$\Delta M_{2}(\boldsymbol{p}, \boldsymbol{q})=G^{2} \pi^{2}|\boldsymbol{q}|^{3} m_{2}^{3}\left[4 \lambda_{1}+\frac{\eta_{1}}{32}\left(11-30 \sigma^{2}+35 \sigma^{4}\right)\right]$,

where $\boldsymbol{p}$ and $\boldsymbol{q}$ are the center-of-mass three-momentum and three-momentum transfer, respectively.

At two loops, the calculation is substantially more complicated due to the proliferation of Feynman diagrams. We refrain from presenting them here explicitly but include them in the Supplemental Material [42] containing the classically truncated integrands. We then apply the exact same integration method discussed at length, in general, 
and in examples in [9]. In this method, the energy components of loop integrals are first localized via residues to matter poles in the potential region of the loop graviton momenta. Afterward, the remaining spatial integrals are expanded to very high order in velocity. Each order then contributes a "bubble" integral, which is evaluated via standard formulas in dimensional regularization [43]. Applying these methods to our two-loop integrands, we obtain integrated expressions up to $\mathcal{O}\left(\boldsymbol{p}^{16}\right)$ in the velocity expansion.

Again following [9], the evaluated integrals exhibit regular patterns that can be resummed to all orders in velocity into a set of simple basis kinematic functions. Resummation is possible because the velocity expansion is done only at the last step, such that the integrands have vestiges of Lorentz invariance, which, together with dimensional analysis and the classical limit, imposes strong constraints on the possible momentum-dependent structures appearing. In particular, the following simple power counting argument shows that new momentum-dependent structures saturate at $\mathcal{O}\left(\boldsymbol{p}^{8}\right)$.
Consider the scattering contribution from $\eta_{i}$. The associated amplitude is dimensionless and proportional to $G^{3} \eta_{i} / m_{i}^{4}$, so the remaining kinematic dependence has mass dimension 12. With a two-loop integral measure and at most seven propagators, the integrand numerator thus has mass dimension 18 at most. (In the standard conventions of amplitudes, we blow up all quartic vertices into factorization diagrams with numerators given by inverse propagators.) A classical contribution requires 7 powers of this mass dimension attributed to loop momenta $\ell$ with the rest attributed to external momenta $p$. This is because the integral measure scales as $\epsilon^{8}$, while the graviton and matter propagators, respectively, scale as $\epsilon^{-8}$ and $\epsilon^{-3}$, so $\epsilon^{7}$ is needed to give a net $\epsilon^{4}$. This all implies a numerator with schematic structure $p^{4}(p \ell)^{7}$, where $p^{4}$ is effectively a constant that can be factored out of the integral. Since the resulting integral produces at most a rank 7 tensor, no new momentum-dependent structures can arise beyond $\mathcal{O}\left(\boldsymbol{p}^{8}\right)$.

In the end, this procedure produces the following twoloop tidal correction to the scattering amplitude:

$$
\begin{aligned}
\Delta M_{3}(\boldsymbol{p}, \boldsymbol{q})= & G^{3} \pi \boldsymbol{q}^{4} \ln |\boldsymbol{q}| m_{2}^{3}\left[4 \lambda_{1}\left(\frac{8 m_{2}}{5}-\frac{m_{1} \sigma\left(5-2 \sigma^{2}\right)}{\left(\sigma^{2}-1\right)^{2}}+\frac{6 m_{1} \sinh ^{-1} \sqrt{\frac{\sigma-1}{2}}}{\left(\sigma^{2}-1\right)^{5 / 2}}\right)+\eta_{1}\left(\frac{m_{2}\left(305-363 \sigma^{2}-110 \sigma^{4}\right)}{560}\right.\right. \\
& \left.-\frac{m_{1} \sigma\left(5401-195 \sigma^{2}-94 \sigma^{4}\right)}{80}-\frac{m_{1} \sigma\left(673+2168 \sigma^{2}\right)}{2\left(\sigma^{2}-1\right)^{2}}+\frac{3 m_{1}\left(33+474 \sigma^{2}+440 \sigma^{4}\right) \sinh ^{-1} \sqrt{\frac{\sigma-1}{2}}}{\left(\sigma^{2}-1\right)^{5 / 2}}\right) \\
& \left.+2\left(1-2 \sigma^{2}\right)\left[4 \lambda_{1}+\frac{\eta_{1}}{32}\left(11-30 \sigma^{2}+35 \sigma^{4}\right)\right] \frac{E\left(E_{2}-m_{2}\right)}{m_{2}\left(\sigma^{2}-1\right)}\right]+\frac{1}{E} \int \frac{d^{3} \ell}{(2 \pi)^{3}} \frac{M_{1}(\boldsymbol{p}, \ell) \Delta M_{2}(\boldsymbol{p}, \ell+\boldsymbol{q})}{\left(\ell^{2}+2 \boldsymbol{p} \ell\right)},
\end{aligned}
$$

where $E=E_{1}+E_{2}$ is the total energy and the nonrelativistic normalization $1 /\left(4 E_{1} E_{2}\right)$ has not been included.

Equation (5) is reminiscent of the $\mathcal{O}\left(G^{3}\right)$ scattering amplitude for pointlike objects [8,9]. In the $m_{1} \ll m_{2}$ expansion, the first term within each round bracket is dominant and all remaining terms are captured at next-toleading order. This accords with the expected mass dependence of the classical scattering angle [12,44], implying that the full $\mathcal{O}\left(G^{3}\right)$ dynamics are accessible from a first order self-force calculation. By the same logic, the $\mathcal{O}\left(G^{2}\right)$ amplitude is completely fixed by the test-particle limit.

As similarly observed for pointlike compact objects $[8,9]$, the final line in Eq. (5) contains both finite and infrared divergent contributions from the iteration of the one-loop tidal contribution $\Delta M_{2}$ with the tree-level amplitude $M_{1}(\boldsymbol{p}, \boldsymbol{q})=-16 \pi G m_{1}^{2} m_{2}^{2}\left(1-2 \sigma^{2}\right) / \boldsymbol{q}^{2}$.

As another check of our resummation, we take $\Delta M_{3}$ in Eq. (5) and reweight each kinematically independent term by a free coefficient. We find that this ansatz is uniquely fixed to Eq. (5) after taking as input our explicitly integrated amplitude at $\mathcal{O}\left(p^{10}\right)$. Consequently, the match of our integrated results to Eq. (5) at $\mathcal{O}\left(\boldsymbol{p}^{16}\right)$ is a highly nontrivial consistency check.
Matching.-Following the approach of [7], we construct an isotropic gauge EFT Hamiltonian, including pointparticle contributions

$$
H^{\mathrm{EFT}}(\boldsymbol{p}, \boldsymbol{r})=\sum_{i=1,2} \sqrt{\boldsymbol{p}^{2}+m_{i}^{2}}+\sum_{n=1}^{\infty} \frac{G^{n} c_{n}\left(\boldsymbol{p}^{2}\right)}{|\boldsymbol{r}|^{n}},
$$

as well as tidal corrections

$$
\Delta H^{\mathrm{EFT}}(\boldsymbol{p}, \boldsymbol{r})=\sum_{n=2}^{\infty} \frac{G^{n} \Delta c_{n}\left(\boldsymbol{p}^{2}\right)}{|\boldsymbol{r}|^{n+4}},
$$

where $\boldsymbol{p}$ and $\boldsymbol{r}$ are the center-of-mass momentum and distance between bodies. Here $c_{n}\left(\boldsymbol{p}^{2}\right)$ and $\Delta c_{n}\left(\boldsymbol{p}^{2}\right)$ dictate the momentum-dependent interactions at zeroth and first order in the tidal coefficients. Explicit expressions for $c_{n}\left(\boldsymbol{p}^{2}\right)$ can be found in Eq. (10.10) of Ref. [9].

The EFT amplitudes, $M^{\mathrm{EFT}}$ and $\Delta M^{\mathrm{EFT}}$, can be trivially computed via Feynman diagrams within the framework of [7] that was used to obtain all of the results in [9]. There it was also observed by explicit calculation that the EFT amplitude in position space is exactly proportional to the 
local center-of-mass momentum squared $\boldsymbol{p}_{\mathrm{loc}}^{2}(\boldsymbol{p}, \boldsymbol{r})$ as a function of the incoming asymptotic momentum $\boldsymbol{p}$ and the separation $\boldsymbol{r}$ between bodies. Consequently, the EFT amplitude can be extracted algebraically from the classical equations of motion, as was later proven in $[15,18]$. Applying this simpler procedure, we obtain

$$
\Delta M_{2}^{\mathrm{EFT}}(\boldsymbol{p}, \boldsymbol{q})=-\frac{G^{2} \pi^{2}|\boldsymbol{q}|^{3} \Delta c_{2}}{12}
$$

for the $\mathcal{O}\left(G^{2}\right)$ tidal correction to the amplitude and

$\Delta M_{3}^{\mathrm{EFT}}(\boldsymbol{p}, \boldsymbol{q})=\frac{G^{3} \pi \boldsymbol{q}^{4} \ln |\boldsymbol{q}|}{30}\left(\Delta c_{3}-\frac{(1-3 \xi) c_{1} \Delta c_{2}}{E \xi}-2 E \xi\left(c_{1} \Delta c_{2}\right)^{\prime}\right)+4 E \xi \int \frac{d^{3} \ell}{(2 \pi)^{3}} \frac{M_{1}^{\mathrm{EFT}}(\boldsymbol{p}, \ell) \Delta M_{2}^{\mathrm{EFT}}(\boldsymbol{p}, \ell+\boldsymbol{q})}{\left(\ell^{2}+2 \boldsymbol{p} \ell\right)}$

at $\mathcal{O}\left(G^{3}\right)$, where $\xi=E_{1} E_{2} /\left(E_{1}+E_{2}\right)^{2}$ and primed differentiation is performed with respect to $\boldsymbol{p}^{2}$. Here we have written the infrared divergent contribution in terms of the iteration of the one-loop $\mathcal{O}\left(G^{2}\right)$ EFT amplitude $\Delta M_{2}^{\mathrm{EFT}}$ together with the $\mathcal{O}(G)$ point-particle EFT amplitude
$M_{1}^{\mathrm{EFT}}(\boldsymbol{p}, \boldsymbol{q})=-4 \pi G c_{1}\left(\boldsymbol{p}^{2}\right) / \boldsymbol{q}^{2}$. Equating these EFT amplitudes to those in the full theory with nonrelativistic normalization factor $1 / 4 E_{1} E_{2}$, we obtain the tidal corrections to the conservative Hamiltonian,

$$
\begin{aligned}
\Delta c_{2}= & -\frac{3 m_{2}^{3}}{E^{2} \xi}\left[4 \lambda_{1}+\frac{\eta_{1}}{32}\left(11-30 \sigma^{2}+35 \sigma^{4}\right)\right], \\
\Delta c_{3}= & \frac{15 m_{2}^{3}}{2 E^{2} \xi}\left[4 \lambda_{1}\left(\frac{8 m_{2}}{5}-\frac{m_{1} \sigma\left(5-2 \sigma^{2}\right)}{\left(\sigma^{2}-1\right)^{2}}+\frac{6 m_{1} \sinh ^{-1} \sqrt{\frac{\sigma-1}{2}}}{\left(\sigma^{2}-1\right)^{5 / 2}}\right)+\eta_{1}\left(\frac{m_{2}\left(305-363 \sigma^{2}-110 \sigma^{4}\right)}{560}\right.\right. \\
& \left.-\frac{m_{1} \sigma\left(5401-195 \sigma^{2}-94 \sigma^{4}\right)}{80}-\frac{m_{1} \sigma\left(673+2168 \sigma^{2}\right)}{2\left(\sigma^{2}-1\right)^{2}}+\frac{3 m_{1}\left(33+474 \sigma^{2}+440 \sigma^{4}\right) \sinh ^{-1} \sqrt{\frac{\sigma-1}{2}}}{\left(\sigma^{2}-1\right)^{5 / 2}}\right) \\
& \left.+2\left(1-2 \sigma^{2}\right)\left[4 \lambda_{1}+\frac{\eta_{1}}{32}\left(11-30 \sigma^{2}+35 \sigma^{4}\right)\right] \frac{E\left(E_{2}-m_{2}\right)}{m_{2}\left(\sigma^{2}-1\right)}\right] \\
& +\frac{3 \nu m_{2}^{3}}{m \gamma^{5} \xi^{3}}\left[\nu(1-\xi)\left(1-2 \sigma^{2}\right)\left(4 \lambda_{1}+\frac{\eta_{1}}{32}\left(11-30 \sigma^{2}+35 \sigma^{4}\right)\right)+4 \gamma^{2} \xi \sigma\left(4 \lambda_{1}+\frac{\eta_{1}}{32}\left(26-95 \sigma^{2}+105 \sigma^{4}\right)\right)\right],
\end{aligned}
$$

where $\gamma=E / m$ and $m=\left(m_{1}+m_{2}\right)$. As anticipated, the infrared divergent contributions to $\Delta M$ and $\Delta M^{\mathrm{EFT}}$ cancel exactly, which is itself a consistency check.

Worldline action.-It will be useful to recast our expressions in terms of the standard notation for the tidal moments in the worldline formalism. The action is given by the point-particle contribution $S^{\mathrm{WL}}=-\sum_{i=1,2} m_{i} \int d \tau_{i}$ together with the leading tidal corrections,

$\Delta S^{\mathrm{WL}}=\sum_{i=1,2} \int d \tau_{i}\left(\frac{\mu_{i}^{(2)}}{4}\left(E_{\alpha \beta}^{i}\right)^{2}+\frac{2 \sigma_{i}^{(2)}}{3}\left(B_{\alpha \beta}^{i}\right)^{2}\right)$.

Here the mass and current quadrupole moments are parametrized in the conventions of Refs. [34,36]. The gravitoelectric and gravitomagnetic tensors $E_{\alpha \beta}^{i}$ and $B_{\alpha \beta}^{i}$ are related to the Weyl curvature invariants evaluated on each worldline by $\left(C_{\alpha \beta \gamma \delta}\right)^{2}=8\left(E_{\alpha \beta}^{i}\right)^{2}-8\left(B_{\alpha \beta}^{i}\right)^{2}$ and $\left(u_{i}^{\mu} u_{i}^{\nu} C_{\mu \alpha \nu \beta}\right)^{2}=$ $\left(E_{\alpha \beta}^{i}\right)^{2}$, where the four velocity $u_{i}$ of each particle satisfies $u_{i}^{2}=-1$. By equating Eq. (11) for a point particle at rest to
Eq. (2) for a static point source for the field, we derive the following relation between the tidal coefficients:

$$
\frac{\lambda_{i}}{m_{i}}=-\frac{\sigma_{i}^{(2)}}{3} \quad \text { and } \quad \frac{\eta_{i}}{m_{i}}=\mu_{i}^{(2)}+\frac{8 \sigma_{i}^{(2)}}{3} .
$$

There is no ambiguity at higher orders in velocity since Eqs. (2) and (11) encode all possible coordinate invariant tidal operators at leading order in derivatives of the metric. Our result for the leading order tidal correction described by $\Delta c_{2}$ exactly matches the result in Eq. (5.13) of Ref. [38], which was derived using a PM worldline effective theory.

Hamiltonian and binding energy.-Our Hamiltonian is consistent with all existing results at the relevant overlapping 2PN accuracy. To show this we transform the tidal Lagrangian in Eq. (5.4) of [34] to a Hamiltonian, as usual taking special care to eliminate acceleration terms and account for the induced shift in coordinates. Again using the EFT methods of [7], we then compute the tidal 
corrections to scattering from this Hamiltonian and find exact agreement with the 2PN terms in Eqs. (4) and (5), i.e., the terms at $\mathcal{O}\left(G^{2} v^{4}\right)$ and $\mathcal{O}\left(G^{3} v^{2}\right)$ and lower. We thus conclude that the $2 \mathrm{PN}$ truncation of our tidal Hamiltonian in Eq. (10) is gauge equivalent to existing results.

As an additional, albeit redundant, check, we use Eq. (10) to compute the tidal corrections to the 1PN circular binding energy and find exact agreement with Eq. (6.5) of [34].

Scattering angle.-Another gauge invariant physical quantity we can compute is the conservative contribution to the classical scattering angle. In Ref. [8], it was observed that this is directly related to the finite parts of the scattering amplitude, and this structure has now been understood to all orders $[15,18]$. The tidal correction to the scattering angle through next-to-leading order is

$$
2 \pi \Delta \chi=\frac{45 p^{4} \Delta \tilde{M}_{2}}{4 E J^{6}}-\frac{96 p^{5} \Delta \tilde{M}_{3}}{E J^{7}}+\frac{12 p^{3} \tilde{M}_{1} \Delta \tilde{M}_{2}}{E^{2} J^{7} \pi^{2}},
$$

where tilded quantities denote finite parts of the corresponding relativistically normalized amplitude contributions with the $\boldsymbol{q}$ dependence stripped off; i.e., $\Delta \tilde{M}_{2}=\Delta M_{2} /|\boldsymbol{q}|^{3}, \quad \Delta \tilde{M}_{3}=\Delta M_{3} /\left(\boldsymbol{q}^{4} \ln |\boldsymbol{q}|\right)$, and $\tilde{M}_{1}=$ $M_{1} q^{2}$, where $M_{1}$ is the point-particle amplitude from
Born exchange defined earlier. We have checked that the $\mathcal{O}\left(J^{-6}\right)$ contribution agrees with Eq. (6.2) of [37].

Test-particle limit.-Our expressions are valid in the testparticle limit. Consider the case of a neutron star orbiting a supermassive black hole. In the strict $m_{1} \ll m_{2}$ limit, particle 1 is effectively a nonminimally coupled test mass residing on a background Schwarzschild spacetime sourced by particle 2. Following the approach of [33], the geodesic trajectory for particle 1 is dictated by the mass shell condition on its four-momentum $p$,

$$
0=p^{\mu} p_{\mu}+m_{1}^{2}-\frac{\lambda_{1}}{2} C_{\alpha \beta \gamma \delta}^{2}-\frac{\eta_{1}}{2 m_{1}^{4}}\left(p^{\mu} p^{\nu} C_{\mu \alpha \nu \beta}\right)^{2},
$$

where all metric contractions are performed with the Schwarzschild metric $g_{\mu \nu}$, taken here to be in isotropic coordinates. We then identify the energy component $p_{0}=$ $H^{\mathrm{Sch}}+\Delta H^{\mathrm{Sch}}$ with the test-particle Hamiltonian including point-particle contributions [45]

$$
H^{\mathrm{Sch}}(\boldsymbol{p}, \boldsymbol{r})=f_{-}(\boldsymbol{r}) f_{+}(\boldsymbol{r})^{-3}\left[\boldsymbol{p}^{2}+f_{+}(\boldsymbol{r})^{4} m_{1}^{2}\right]^{1 / 2},
$$

as well as corrections linear in the tidal coefficients,

$$
\begin{aligned}
\Delta H^{\operatorname{Sch}}(\boldsymbol{p}, \boldsymbol{r})= & 3 \lambda_{1}\left(-\frac{R^{2}}{\boldsymbol{r}^{6} E_{1}}+\frac{R^{3}\left(6 \boldsymbol{p}^{2}+7 m_{1}^{2}\right)}{2|\boldsymbol{r}|^{7} E_{1}^{3}}\right) \\
& +\frac{3 \eta_{1}}{8}\left(-\frac{R^{2}\left(1+3 \boldsymbol{v}_{\perp}^{2}+3 \boldsymbol{v}_{\perp}^{4}\right)}{\boldsymbol{r}^{6} E_{1}}+\frac{R^{3}\left[\boldsymbol{p}^{2}\left(6+24 \boldsymbol{v}_{\perp}^{2}+30 \boldsymbol{v}_{\perp}^{4}\right)+m_{1}^{2}\left(7+27 \boldsymbol{v}_{\perp}^{2}+33 \boldsymbol{v}_{\perp}^{4}\right)\right]}{2|\boldsymbol{r}|^{7} E_{1}^{3}}\right)+\mathcal{O}\left(\boldsymbol{r}^{-8}\right),
\end{aligned}
$$

where $f_{ \pm}(\boldsymbol{r})=1 \pm(R / 4|\boldsymbol{r}|), R=2 G m_{2}$ is the Schwarzschild radius, and $\boldsymbol{v}_{\perp}^{2}=\left(\boldsymbol{p}^{2}-\left[(\boldsymbol{p} \cdot \boldsymbol{r})^{2} / \boldsymbol{r}^{2}\right]\right) / m_{1}^{2}$. Note that, for $m_{1} \ll m_{2}$, Eq. (15) is valid to all orders in $\boldsymbol{p}$ and $\boldsymbol{r}$, while Eq. (16) is truncated at $\mathcal{O}\left(|\boldsymbol{r}|^{-7}\right)$ for the sake of brevity.

It is easy to see that the terms in $\Delta H^{\text {Sch }}$ proportional to $\lambda_{1}$ at $\mathcal{O}\left(\boldsymbol{r}^{-6}\right)$ and $\mathcal{O}\left(|\boldsymbol{r}|^{-7}\right)$ coincide exactly with the $m_{1} \ll m_{2}$ limit of $\Delta c_{2}$ and $\Delta c_{3}$ in Eq. (10). On the other hand, a comparison of the $\eta_{1}$ corrections is not so straightforward since the relevant terms in $\Delta H^{\text {Sch }}$ depend on $\boldsymbol{p} \cdot \boldsymbol{r}$ and thus depart from the isotropic gauge of Eq. (10). Hence, a proper comparison requires constructing a canonical transformation between gauges or, alternatively, computing a physical, gauge invariant quantity such as the scattering amplitude. Using the EFT approach of [7], we compute the $\eta_{1}$ tidal corrections to the scattering amplitude and find an exact match to the $m_{1} \ll m_{2}$ limit of Eqs. (4) and (5) after including nonrelativistic normalization. This match between scattering amplitudes implies that the test-particle limit of our Hamiltonian is gauge equivalent to Eq. (16).

Discussion.-We have presented the first ever calculation of tidal corrections to the conservative Hamiltonian for spinless compact objects at next-to-leading order in the PM expansion. These dynamics are extracted from the two-loop $\mathcal{O}\left(G^{3}\right)$ scattering amplitude at linear order in the mass and current quadrupole moments.

Our expressions pass many checks. Still, it would be interesting to verify them with traditional methods, e.g., as was done for the point-particle 3PM Hamiltonian [8,9] at 5PN and 6PN via self-force theory [46] and PN perturbation theory [47]. Also valuable would be a comparison of our results against other approaches, like numerical relativity and effective one-body formalism, as was done in [48] for the case of the binary black hole inspiral.

We are grateful to Zvi Bern, Luc Blanchet, Thibault Damour, Walter Goldberger, Ira Rothstein, Jan Steinhoff, and Justin Vines for comments on this manuscript. C. C. and M.P.S. are supported by the DOE under Award No. DE-SC0011632 and by the Walter Burke Institute for Theoretical Physics. The calculations here used the computer algebra system Mathematica [49] in combination with FeynCalc [50] and $\mathrm{X}_{\text {Act }}$ [51], as well as the On-Line Encyclopedia of Integer Sequences [52] and the Hoffman2 
Cluster at the Institute for Digital Research and Education at UCLA.

[1] B. P. Abbottetal. (LIGOScientific and Virgo Collaborations), Phys. Rev. Lett. 116, 061102 (2016); 119, 161101 (2017).

[2] Y. Iwasaki, Prog. Theor. Phys. 46, 1587 (1971); Lett. Nuovo Cimento 1, 783 (1971); Lett. Nuovo Cimento 1, 783 (1971)]; H. Okamura, T. Ohta, T. Kimura, and K. Hiida, Prog. Theor. Phys. 50, 2066 (1973); D. Amati, M. Ciafaloni, and G. Veneziano, Nucl. Phys. B347, 550 (1990); J. F. Donoghue, Phys. Rev. Lett. 72, 2996 (1994); Phys. Rev. D 50, 3874 (1994); N. E. J. Bjerrum-Bohr, J. F. Donoghue, and B. R. Holstein, Phys. Rev. D 67, 084033 (2003); 71, 069903 (E) (2005); B. R. Holstein and A. Ross, arXiv:0802.0716; N. E. J. Bjerrum-Bohr, J. F. Donoghue, and P. Vanhove, J. High Energy Phys. 02 (2014) 111.

[3] W. D. Goldberger and I. Z. Rothstein, Phys. Rev. D 73, 104029 (2006).

[4] H. Kawai, D. C. Lewellen, and S. H. H. Tye, Nucl. Phys. B269, 1 (1986); Z. Bern, L. J. Dixon, M. Perelstein, and J. S. Rozowsky, Nucl. Phys. B546, 423 (1999); Z. Bern, John Joseph M. Carrasco, and H. Johansson, Phys. Rev. D 78, 085011 (2008); Phys. Rev. Lett. 105, 061602 (2010); Z. Bern, J. J. Carrasco, M. Chiodaroli, H. Johansson, and R. Roiban, arXiv:1909.01358.

[5] Z. Bern, L. J. Dixon, D. C. Dunbar, and D. A. Kosower, Nucl. Phys. B425, 217 (1994); B435, 59 (1995); Z. Bern, L. J. Dixon, and D. A. Kosower, Nucl. Phys. B513, 3 (1998); R. Britto, F. Cachazo, and B. Feng, Nucl. Phys. B725, 275 (2005); Z. Bern, J. J. M. Carrasco, H. Johansson, and D. A. Kosower, Phys. Rev. D 76, 125020 (2007).

[6] D. Neill and I. Z. Rothstein, Nucl. Phys. B877, 177 (2013).

[7] C. Cheung, I. Z. Rothstein, and M. P. Solon, Phys. Rev. Lett. 121, 251101 (2018).

[8] Z. Bern, C. Cheung, R. Roiban, C. H. Shen, M. P. Solon, and M. Zeng, Phys. Rev. Lett. 122, 201603 (2019).

[9] Z. Bern, C. Cheung, R. Roiban, C. H. Shen, M. P. Solon, and M. Zeng, J. High Energy Phys. 10 (2019) 206.

[10] C. Cheung and M. P. Solon, J. High Energy Phys. 06 (2020) 144.

[11] V. Vaidya, Phys. Rev. D 91, 024017 (2015); A. Guevara, J. High Energy Phys. 04 (2019) 033; N. Arkani-Hamed, T. C. Huang, and Y.t. Huang, arXiv:1709.04891; J. Vines, Classical Quantum Gravity 35, 084002 (2018); A. Guevara, A. Ochirov, and J. Vines, J. High Energy Phys. 09 (2019) 056; M. Z. Chung, Y. T. Huang, J. W. Kim, and S. Lee, J. High Energy Phys. 04 (2019) 156; Y. F. Bautista and A. Guevara, arXiv:1903.12419; B. Maybee, D. O'Connell, and J. Vines, J. High Energy Phys. 12 (2019) 156; A. Guevara, A. Ochirov, and J. Vines, Phys. Rev. D 100, 104024 (2019); N. Arkani-Hamed, Y.t. Huang, and D. O'Connell, J. High Energy Phys. 01 (2020) 046; M. Z. Chung, Y. T. Huang, and J. W. Kim, J. High Energy Phys. 09 (2020) 074; arXiv:1911.12775; M. Levi, A. J. Mcleod, and M. Von Hippel, arXiv:2003.02827; M. Z. Chung, Y. t. Huang, J. W. Kim, and S. Lee, J. High Energy Phys. 05 (2020) 105; M. Levi, A. J. Mcleod, and M. Von Hippel, arXiv:2003.07890; Z. Bern, A. Luna, R. Roiban, C. H. Shen, and M. Zeng, arXiv:2005.03071.
[12] J. Vines, J. Steinhoff, and A. Buonanno, Phys. Rev. D 99, 064054 (2019).

[13] S. Caron-Huot and Z. Zahraee, J. High Energy Phys. 07 (2019) 179; J. Parra-Martinez, M. S. Ruf, and M. Zeng, arXiv:2005.04236.

[14] J. M. Henn and B. Mistlberger, J. High Energy Phys. 05 (2019) 023; P. Di Vecchia, S. G. Naculich, R. Russo, G. Veneziano, and C. D. White, J. High Energy Phys. 03 (2020) 173; Z. Bern, H. Ita, J. Parra-Martinez, and M. S. Ruf, Phys. Rev. Lett. 125, 031601 (2020); S. Abreu, F. Febres Cordero, H. Ita, M. Jaquier, B. Page, M. S. Ruf, and V. Sotnikov, Phys. Rev. Lett. 124, 211601 (2020).

[15] A. Cristofoli, N. E. J. Bjerrum-Bohr, P. H. Damgaard, and P. Vanhove, Phys. Rev. D 100, 084040 (2019); N. E. J. BjerrumBohr, A. Cristofoli, and P. H. Damgaard, J. High Energy Phys. 08 (2020) 038; A. Cristofoli, P. H. Damgaard, P. Di Vecchia, and C. Heissenberg, J. High Energy Phys. 07 (2020) 122.

[16] P. H. Damgaard, K. Haddad, and A. Helset, J. High Energy Phys. 11 (2019) 070; R. Aoude, K. Haddad, and A. Helset, J. High Energy Phys. 05 (2020) 051; K. Haddad and A. Helset, Phys. Rev. Lett. 125, 181603 (2020).

[17] A. Luna, R. Monteiro, I. Nicholson, D. O'Connell, and C. D. White, J. High Energy Phys. 06 (2016) 023; W. D. Goldberger and A. K. Ridgway, Phys. Rev. D 95, 125010 (2017); W. D. Goldberger, S. G. Prabhu, and J. O. Thompson, Phys. Rev. D 96, 065009 (2017); F. Cachazo and A. Guevara, J. High Energy Phys. 02 (2020) 181; W. D. Goldberger and A. K. Ridgway, Phys. Rev. D 97, 085019 (2018); D. Chester, Phys. Rev. D 97, 084025 (2018); W. D. Goldberger, J. Li, and S. G. Prabhu, Phys. Rev. D 97, 105018 (2018); J. Li and S. G. Prabhu, Phys. Rev. D 97, 105019 (2018); A. Laddha and A. Sen, Phys. Rev. D 100, 024009 (2019); N. E. J. Bjerrum-Bohr, P. H. Damgaard, G. Festuccia, L. Planté, and P. Vanhove, Phys. Rev. Lett. 121, 171601 (2018); C. H. Shen, J. High Energy Phys. 11 (2018) 162; J. Plefka, J. Steinhoff, and W. Wormsbecher, Phys. Rev. D 99, 024021 (2019); B. Sahoo and A. Sen, J. High Energy Phys. 02 (2019) 086; D. A. Kosower, B. Maybee, and D. O'Connell, J. High Energy Phys. 02 (2019) 137; M. Ciafaloni, D. Colferai, and G. Veneziano, Phys. Rev. D 99, 066008 (2019); A. Koemans Collado, P. Di Vecchia, and R. Russo, Phys. Rev. D 100, 066028 (2019).

[18] G. Kälin and R. A. Porto, J. High Energy Phys. 01 (2020) 072; G. Kälin and R. A. Porto, J. High Energy Phys. 02 (2020) 120.

[19] A. Buonanno and T. Damour, Phys. Rev. D 59, 084006 (1999); Phys. Rev. D 62, 064015 (2000).

[20] F. Pretorius, Phys. Rev. Lett. 95, 121101 (2005); M. Campanelli, C. O. Lousto, P. Marronetti, and Y. Zlochower, Phys. Rev. Lett. 96, 111101 (2006); J. G. Baker, J. Centrella, D. I. Choi, M. Koppitz, and J. van Meter, Phys. Rev. Lett. 96, 111102 (2006).

[21] Y. Mino, M. Sasaki, and T. Tanaka, Phys. Rev. D 55, 3457 (1997); T. C. Quinn and R. M. Wald, Phys. Rev. D 56, 3381 (1997).

[22] J. Droste. Proc. Natl. Acad. Sci. U.S.A. 19, 447455 (1916); A. Einstein, L. Infeld, and B. Hoffmann, Ann. Math. 39, 65 (1938); T. Ohta, H. Okamura, T. Kimura, and K. Hiida, Prog. Theor. Phys. 50, 492 (1973); P. Jaranowski and G. Schäfer, Phys. Rev. D 57, 7274 (1998); 63, 029902(E) 
(2000); T. Damour, P. Jaranowski, and G. Schäfer, Phys. Rev. D 62, 044024 (2000); L. Blanchet and G. Faye, Phys. Lett. A 271, 58 (2000); T. Damour, P. Jaranowski, and G. Schäfer, Phys. Lett. B 513, 147 (2001); T. Damour, P. Jaranowski, and G. Schäfer, Phys. Rev. D 89, 064058 (2014); P. Jaranowski and G. Schäfer, Phys. Rev. D 92, 124043 (2015); L. Bernard, L. Blanchet, A. Boh, G. Faye, and S. Marsat, Phys. Rev. D 93, 084037 (2016); L. Bernard, L. Blanchet, A. Boh, G. Faye, and S. Marsat, Phys. Rev. D 95, 044026 (2017); D. Bini and T. Damour, Phys. Rev. D 96, 064021 (2017); L. Bernard, L. Blanchet, A. Boh, G. Faye, and S. Marsat, Phys. Rev. D 96, 104043 (2017); T. Marchand, L. Bernard, L. Blanchet, and G. Faye, Phys. Rev. D 97, 044023 (2018); L. Bernard, L. Blanchet, G. Faye, and T. Marchand, Phys. Rev. D 97, 044037 (2018).

[23] B. Bertotti, Nuovo Cimento 4, 898 (1956); R. P. Kerr, Nuovo Cimento (1955-1965) 13, 469491 (1959); B. Bertotti and J. F. Plebański, Ann. Phys. (Leipzig) 11, 169200 (1960); M. Portilla, J. Phys. A 12, 1075 (1979); K. Westpfahl and M. Goller, Lett. Nuovo Cimento 26, 573 (1979); M. Portilla, J. Phys. A 13, 3677 (1980); L. Bel, T. Damour, N. Deruelle, J. Ibanez, and J. Martin, Gen. Relativ. Gravit. 13, 963 (1981); K. Westpfahl, Fortschr. Phys. 33, 417 (1985); T. Ledvinka, G. Schaefer, and J. Bicak, Phys. Rev. Lett. 100, 251101 (2008); T. Damour, Phys. Rev. D 94, 104015 (2016); 97, 044038 (2018).

[24] J. B. Gilmore and A. Ross, Phys. Rev. D 78, 124021 (2008); S. Foffa and R. Sturani, Phys. Rev. D 84, 044031 (2011); S. Foffa, P. Mastrolia, R. Sturani, and C. Sturm, Phys. Rev. D 95, 104009 (2017); R. A. Porto and I. Z. Rothstein, Phys. Rev. D 96, 024062 (2017); S. Foffa, P. Mastrolia, R. Sturani, C. Sturm, and W. J. Torres Bobadilla, Phys. Rev. Lett. 122, 241605 (2019); J. Blümlein, A. Maier, and P. Marquard, Phys. Lett. B 800, 135100 (2020); S. Foffa, R. A. Porto, I. Rothstein, and R. Sturani, Phys. Rev. D 100, 024048 (2019); J. Blümlein, A. Maier, P. Marquard, and G. Schäfer, Nucl. Phys. B955, 115041 (2020).

[25] A. Buonanno and B. Sathyaprakash, arXiv:1410.7832.

[26] T. Dietrich, T. Hinderer, and A. Samajdar, arXiv: 2004.02527.

[27] B. P. Abbott et al. (LIGO Scientific and Virgo Collaborations), Phys. Rev. Lett. 119, 161101 (2017).

[28] B. P. Abbott et al. (LIGO Scientific and Virgo Collaborations), Astrophys. J. Lett. 892, L3 (2020).

[29] E. E. Flanagan and T. Hinderer, Phys. Rev. D 77, 021502(R) (2008).

[30] C. Cutler, T. A. Apostolatos, L. Bildsten, L. S. Finn, E. E. Flanagan, D. Kennefick, D. M. Markovic, A. Ori, E. Poisson, G. J. Sussman, and K. S. Thorne, Phys. Rev. Lett. 70, 2984 (1993); C. Cutler and E. E. Flanagan, Phys. Rev. D 49, 2658 (1994); T. Hinderer, B. D. Lackey, R. N. Lang, and J. S. Read, Phys. Rev. D 81, 123016 (2010); F. Pannarale, L. Rezzolla, F. Ohme, and J. S. Read, Phys. Rev. D 84, 104017 (2011); T. Damour, A. Nagar, and L. Villain, Phys. Rev. D 85, 123007 (2012); M. Favata, Phys. Rev. Lett. 112, 101101 (2014); K. Yagi and N. Yunes, Phys. Rev. D 89, 021303 (2014); B. D. Lackey and L. Wade, Phys. Rev. D 91, 043002 (2015); L. Wade, J. D. Creighton, E. Ochsner, B. D. Lackey, B. F. Farr, T. B. Littenberg, and V. Raymond, Phys. Rev. D 89, 103012 (2014); K. Yagi and N. Yunes, Classical
Quantum Gravity 34, 015006 (2017); S. E. Gralla, Classical Quantum Gravity 35, 085002 (2018); A. Samajdar and T. Dietrich, Phys. Rev. D 98, 124030 (2018); E. Poisson, Phys. Rev. D 101, 104028 (2020).

[31] M. Shibata, Phys. Rev. D 60, 104052 (1999); L. Baiotti, B. Giacomazzo, and L. Rezzolla, Phys. Rev. D 78, 084033 (2008); S. Bernuzzi, A. Nagar, M. Thierfelder, and B. Brugmann, Phys. Rev. D 86, 044030 (2012); S. Bernuzzi, A. Nagar, T. Dietrich, and T. Damour, Phys. Rev. Lett. 114, 161103 (2015); J. S. Read, C. Markakis, M. Shibata, K. Uryu, J. D. Creighton, and J. L. Friedman, Phys. Rev. D 79, 124033 (2009); L. Baiotti, T. Damour, B. Giacomazzo, A. Nagar, and L. Rezzolla, Phys. Rev. Lett. 105, 261101 (2010); L. Baiotti, T. Damour, B. Giacomazzo, A. Nagar, and L. Rezzolla, Phys. Rev. D 84, 024017 (2011); B. D. Lackey, K. Kyutoku, M. Shibata, P. R. Brady, and J. L. Friedman, Phys. Rev. D 85, 044061 (2012).

[32] T. Damour, M. Soffel, and C. Xu, Phys. Rev. D 47, 3124 (1993); Phys. Rev. D 49, 618 (1994); T. Hinderer, Astrophys. J. 677, 1216 (2008); T. Damour and A. Nagar, Phys. Rev. D 80, 084035 (2009); T. Binnington and E. Poisson, Phys. Rev. D 80, 084018 (2009); J. E. Vines and E. E. Flanagan, Phys. Rev. D 88, 024046 (2013); J. Vines, E. E. Flanagan, and T. Hinderer, Phys. Rev. D 83, 084051 (2011); Q. Henry, G. Faye, and L. Blanchet, Phys. Rev. D 102, 044033 (2020).

[33] J. Steinhoff, T. Hinderer, A. Buonanno, and A. Taracchini, Phys. Rev. D 94, 104028 (2016).

[34] Q. Henry, G. Faye, and L. Blanchet, Phys. Rev. D 101, 064047 (2020).

[35] D. Bini and T. Damour, Phys. Rev. D 90, 124037 (2014); C. Kavanagh, A. C. Ottewill, and B. Wardell, Phys. Rev. D 92 , 084025 (2015); P. Nolan, C. Kavanagh, S. R. Dolan, A. C. Ottewill, N. Warburton, and B. Wardell, Phys. Rev. D 92 , 123008 (2015).

[36] T. Damour and A. Nagar, Phys. Rev. D 81, 084016 (2010); D. Bini, T. Damour, and G. Faye, Phys. Rev. D 85, 124034 (2012).

[37] D. Bini, T. Damour, and A. Geralico, Phys. Rev. D 101, 044039 (2020).

[38] G. Kälin and R. A. Porto, arXiv:2006.01184.

[39] M. Punturo et al., Classical Quantum Gravity 27, 194002 (2010); M. Maggiore, C. Van Den Broeck, N. Bartolo, E. Belgacem, D. Bertacca, M. A. Bizouard, M. Branchesi, S. Clesse, S. Foffa, J. Garca-Bellido, S. Grimm, J. Harms, T. Hinderer, S. Matarrese, C. Palomba, M. Peloso, A. Ricciardone, and M. Sakellariadou, J. Cosmol. Astropart. Phys. 03 (2020) 050.

[40] D. Lovelock, Math. Proc. Cambridge Philos. Soc. 68, 345 (1970); S. B. Edgar and A. Hoglund, J. Math. Phys. (N.Y.) 43, 659 (2002).

[41] C. Cheung and G. N. Remmen, J. High Energy Phys. 01 (2017) 104; C. Cheung and G. N. Remmen, J. High Energy Phys. 09 (2017) 002.

[42] See Supplemental Material at http://link.aps.org/ supplemental/10.1103/PhysRevLett.125.191601 for the set of two-loop integrands in deDonder gauge for extracting the tidal corrections to the classical potential.

[43] V. A. Smirnov, Springer Tracts Mod. Phys. 250, 1 (2012).

[44] T. Damour, Phys. Rev. D 102, 024060 (2020). 
[45] N. Wex and G. Schäfer, Classical Quantum Gravity 10, 2729 (1993).

[46] D. Bini, T. Damour, and A. Geralico, Phys. Rev. Lett. 123, 231104 (2019); 102, 024062 (2020); 102, 024061 (2020).

[47] J. Blmlein, A. Maier, P. Marquard, and G. Schfer, Phys. Lett. B 807, 135496 (2020).

[48] A. Antonelli, A. Buonanno, J. Steinhoff, M. van de Meent, and J. Vines, Phys. Rev. D 99, 104004 (2019).

[49] Wolfram Research, Inc., Mathematica, Version 12.0, Champaign, IL (2019), https://www.wolfram.com/mathematica/.
[50] R. Mertig, M. Bohm, and A. Denner, Comput. Phys. Commun. 64, 345 (1991); V. Shtabovenko, R. Mertig, and F. Orellana, Comput. Phys. Commun. 207, 432 (2016); V. Shtabovenko, R. Mertig, and F. Orellana, Comput. Phys. Commun. 256, 107478 (2020).

[51] Jose M. Martin-Garcia et al., xAct: Efficient tensor computer algebra for the Wolfram Language http://www.xact.es/.

[52] OEIS Foundation Inc., The On-Line Encyclopedia of Integer Sequences (2020) http://oeis.org. 